INPLASY

PROTOCOL

To cite: He et al. The effectiveness and safety of Chinese herbal formulas on skin photoaging : A protocol for systematic review and meta-analysis. Inplasy protocol 2020120005. doi:

10.37766/inplasy2020.12.0005

Received: 01 December 2020

Published: 01 December 2020

Corresponding author: Jingdong Wu

Inzywjd@163.com

Author Affiliation: Liaoning University of Traditional Chinese Medicine Hospital 2nd Affiliated Hospital

Support: KPNSLP

(NO.20170540605).

Review Stage at time of this submission: Preliminary searches.

Conflicts of interest: No.

\section{The effectiveness and safety of Chinese herbal formulas on skin photoaging : A protocol for systematic review and meta-analysis}

He, J1; Wu, J2; Lv, L3; Zhang, X4; Zhang,Y55.

Review question / Objective: This systematic review and meta-analysis aims to evaluate the effectiveness and safety of Chinese herbal formulas(CHFs) alone or in combination with conventional western medicine measures in the treatment of skin photoaging.

Condition being studied: Skin photoaging (SP) is a chronic inflammatory process induced by sunlight, especially ultraviolet radiation. In recent years, the number of randomized controlled trials (RCTs) of CHFs on skin photoaging is increasing gradually, but there is no systematic review to synthesize these results of RCTs.

Information sources: We will search 9 electronic literature databases (PubMed, EMBASE, Web of Science, CENTRAL, CINAHL, CBM, CNKI, CQVIP and WF) for RCTs. There is no restriction on the language of publication.

INPLASY registration number: This protocol was registered with the International Platform of Registered Systematic Review and Meta-Analysis Protocols (INPLASY) on 01 December 2020 and was last updated on 01 December 2020 (registration number INPLASY2020120005).

\section{INTRODUCTION}

Review question / Objective: This systematic review and meta-analysis aims to evaluate the effectiveness and safety of Chinese herbal formulas(CHFs) alone or in combination with conventional western medicine measures in the treatment of skin photoaging.

Condition being studied: Skin photoaging (SP) is a chronic inflammatory process induced by sunlight, especially ultraviolet radiation. In recent years, the number of randomized controlled trials (RCTs) of 
CHFs on skin photoaging is increasing gradually, but there is no systematic review to synthesize these results of RCTs.

\section{METHODS}

Participant or population: Patients diagnosed as skin photoaging by any guidelines or consensus with clear diagnostic criteria will be included, regardless of age, region, race, country.

Intervention: CHFs alone or combined with conventional western medicine interventions. There will be no limit on the dosage form, frequency and course of treatment.

Comparator: Conventional western medicine measures or no treatment.

Study designs to be included: Literature search, literature screening, data extraction, software analysis and conclusion.

Eligibility criteria: RCTs are limited to Chinese and English.

Information sources: We will search 9 electronic literature databases (PubMed, EMBASE, Web of Science, CENTRAL, CINAHL, CBM, CNKI, CQVIP and WF) for RCTs. There is no restriction on the language of publication.

Main outcome(s): Symptom score (skin relaxation, telangiectasia, pore coarseness, pigmentation, etc.), total effective rate, adverse reactions.

Quality assessment / Risk of bias analysis: Two researchers will use RoB 2.0 tool to assess the quality of the included RCTs, and the AMSTAR-2 tool to systematic reviews. Disagreements will be resolved through discussion or judged by a third senior reviewer.

Strategy of data synthesis: We will perform meta-analysis using stata13.1 software.If available data are insufficient, qualitative synthesis will be performed. The Q-test and 12 values will be used to evaluate inter- study heterogeneity. When the p-value of Q-test $>0.1$ and $12<50 \%$, a fixed-effects model will be applied; otherwise, a random-effects model will be used. Dichotomous data will be presented as odds ratio (OR) with corresponding 95\% confidence intervals (Cls), continuous variables will be calculated as mean difference (MD) with corresponding 95\% Cls.

Subgroup analysis: None planned for the systematic review and meta-analysis.

Sensibility analysis: Based on the evaluation results of RoB 2.0 tool, we will conduct sensitivity analysis by eliminating low-quality study.

Country(ies) involved: China.

Keywords: photoaging, Chinese herb formula, protocol, systematic review.

Contributions of each author:

Author 1 - Jiyong He.

Author 2 - Jingdong Wu.

Author 3 - Ling Lv.

Author 4 - Xiaoqing Zhang.

Author 5 - Yu Zhang. 